Milton Camelo Rincón*

Jacobo Campo Robledo***

Universidad Católica de Colombia, Bogotá, Colombia.

Recibido: 29 de octubre de 2014

Concepto de evaluación: 31 de noviembre de 2015

Aprobado: 9 de diciembre de 2015

Artículo de investigación (C) 2016 Universidad Católica de Colombia. Facultad de Ciencias Económicas y Administrativas. Todos los derechos reservados

* Economista y Magíster en Ciencias Económicas. Docente de tiempo completo, Facultad de Ciencias Económicas y Sociales, Universidad de La Salle, Bogotá, Colombia. Correo electrónico: mscamelo@unisalle.edu.co

** Magíster en Economía, investigador del Grupo Finanzas y Política Económica, y docente de la Facultad de Ciencias Económicas y Administrativas, Universidad Católica de Colombia, Bogotá, Colombia. Dirección de correspondencia: Cra. 13 \# 47 - 49, Facultad de Economía, Bogotá, Colombia. Correo electrónico: jacampo@ucatolica.edu.co

\section{Análisis de la política de vivienda en Bogotá: un enfoque desde la oferta y la demanda*}

\section{RESUMEN}

En este artículo se realiza una revisión de los antecedentes asociados a la política de vivienda y el sector de la construcción en Colombia, desde la década de 1970 hasta los años recientes. Con base en un análisis de oferta y demanda, el texto ofrece una explicación teórica de la problemática actual en el mercado de la vivienda en Bogotá. Así, a partir de la revisión documental y el examen de variables como producción, precios, créditos hipotecarios y área construida, se sugiere que el enfoque basado en promover la demanda de vivienda ha generado efectos nocivos sobre los precios y el desarrollo urbanístico de la ciudad. Como resultado, el artículo sintetiza los principales obstáculos de la consolidación de una política de vivienda sostenible, a la vez que destaca la importancia de considerar políticas alternativas basadas en el mejoramiento y la construcción en sitio propio, con criterios de eficiencia económica y dignidad social.

Palabras clave: política de vivienda, oferta, demanda, sector construcción..

JEL : D11, R31, Z18

\section{An analysis of housing policy in Bogotá: a supply and demand perspective}

\section{ABSTRACT}

This article reviews the background to Colombian housing and construction sector policy from the 1970s to recent times. Based on a supply and demand analysis the text offers a theoretical explanation of the current problems of the housing market in Bogotá. Based on a literature review and

Este documento es resultado de un proyecto de investigación más amplio titulado "Proyecto de vivienda adecuada y sostenible para el barrio La Paz en Bogotá" y desarrollado por investigadores de la Facultad de Arquitectura y la Facultad de Economía de la Universidad Católica de Colombia. Cualquier error u omisión es responsabilidad de los autores y no compromete en ningún momento a la institución. 
an examination of such variables as production, prices, mortgage credits and constructed area, it is suggested that a focus on promoting demand for housing has produced negative effects on prices and on urban development in the city. Consequently, the article synthesizes the principal obstacles to consolidating a sustainable housing policy, while at the same time highlighting the importance of considering alternative policies based on improving existing stock and building in appropriate places, using to criteria designed to guarantee economic efficiency and social dignity.

Keywords: housing policy, supply and demand, construction sector.

\section{Análise da política habitacional em Bogotá: um enfoque a partir da oferta e da demanda}

\section{RESUMO}

Este artigo realiza uma revisão dos antecedentes associados com a política habitacional e o setor da construção na Colômbia, a partir da década de 1970 até os anos recentes. Com base numa análise de oferta e demanda, o texto oferece uma explicação teórica da problemática atual no mercado habitacional em Bogotá. Assim, a partir da revisão de documentos e do exame de variáveis como produção, preços, créditos hipotecários e área construída, sugere-se que o enfoque baseado em promover a demanda de moradia gerou efeitos nocivos sobre os preços e o desenvolvimento urbanístico da cidade. Como resultado, o artigo sintetiza os principais obstáculos da consolidação de uma política habitacional sustentável, ao mesmo tempo em que destaca a importância de considerar políticas alternativas baseadas no melhoramento e na construção em lugar adequado, com critérios de eficiência econômica e dignidade social.

Palavras-chave: política habitacional, oferta e demanda, setor construção. 


\section{INTRODUCCIÓN}

La problemática de la vivienda en Bogotá presenta diversos matices. En muchos sectores de la ciudad son latentes problemas como la ilegalidad, el riesgo de las estructuras, la segregación social y el difícil acceso geográfico y a los servicios sociales básicos. No obstante, actualmente los factores de mayor preocupación son el acelerado crecimiento en los precios y la escasez del suelo.

A pesar de que estos son problemas ampliamente discutidos, la ejecución de soluciones efectivas no se vislumbra en el corto plazo. Desde este precedente, este artículo desarrolla una discusión teórica y factual, con la que se propone un debate orientado a propiciar la oferta de vivienda digna, adecuada y sostenible (Villaveces, 2007).

En este análisis se muestra que las políticas públicas han sido orientadas principalmente a la generación de demanda, en particular, hacia mecanismos de financiamiento de compra de vivienda nueva (Urrutia y Namen, 2012), lo que se asocia estrechamente a la dinámica de financiarización de la economía en las últimas décadas. La mayor parte de estudios académicos y oficiales en torno al mercado de vivienda reflejan dicha orientación; la historia de la política de vivienda casi que se limita a la historia del crédito hipotecario (Jaramillo y Cuervo, 2009; Echeverry, García y Pineda, 1999), mientras que los temas de mejoramiento se develan solo ligeramente.

Ahora bien, surgen ciertos cuestionamientos clave que direccionan este análisis: ¿cuál es la política de vivienda que ha predominado históricamente en Colombia? ¿Cómo ha evolucionado el sector de la construcción ante dichas políticas? ¿Qué otros factores afectan el comportamiento en el mercado de vivienda? ¿Por qué los precios de la vivienda en Bogotá han crecido sin control? Si bien sería ambicioso responder plenamente a estas preguntas, el propósito es dar luces sobre estos cuestionamientos y ofrecer una explicación teórica y, al mismo tiempo, basada en los datos (lo factual), sobre lo acaecido es este mercado durante los últimos años.
Con este propósito, el artículo se organiza en seis secciones, de las que la primera es esta: introducción; la segunda presenta un marco referencial de los avances en investigaciones en el ámbito internacional acerca de los programas de desarrollo de vivienda; en la tercera se realiza una revisión de los antecedentes de la política de vivienda en Colombia y su estrecho vínculo con el sector financiero; en la cuarta se examinan algunos hechos estilizados del sector de la construcción y la evolución de algunas variables centrales del mercado de la vivienda; en la quinta se desarrolla un diagnóstico de la problemática actual y una explicación teórica simplificada del mercado de vivienda, desde el enfoque de equilibrio parcial; en la sexta y última sección se realizan algunas consideraciones finales.

\section{IMPORTANCIA DE LA POLÍTICA DE VIVIENDA A NIVEL INTERNACIONAL}

A continuación se presenta una revisión de las principales tendencias en investigación relacionadas con la política de vivienda en el contexto mundial, con la finalidad de establecer un marco referencial de los avances en investigaciones de corte internacional acerca de los programas de desarrollo de vivienda. Así, se abordarán temas como la relación entre vivienda y la salud física y mental de sus habitantes, vivienda y desigualdad económica, entre otros.

Novoa et al. (2014) presentan un análisis descriptivo acerca del impacto que tiene la crisis económica sobre las condiciones de vivienda en la salud de los habitantes, tanto física como mental. Este impacto se lleva a cabo a través de cuatro dimensiones identificadas por la Organización Mundial de la Salud (OMS) y justificadas por los autores: el hogar, las condiciones físicas de la vivienda, el entorno físico y el entorno social. Exponen como ejemplo el caso de España, cuya situación actual lo ubica en el primer puesto de los países de la Organización para la Cooperación 
y el Desarrollo Económicos (OCDE) con el mayor número de viviendas per cápita.

Por otro lado, en los países africanos se han establecido diez supuestos que son constantes en el diseño de las políticas de vivienda, algunos de los cuales son estos: los arriendos son demasiado altos, la vivienda adecuada depende del nivel de ingreso de los hogares y cada hogar debe convertirse en propietario de vivienda. Tipple (2015), a partir del análisis de casos de siete países de la región del África Subsariana, muestra que los diez supuestos constantes que parecen decidir la formulación de políticas públicas en materia de vivienda en los países africanos no son generalmente ciertos. Este trabajo muestra también que no hay una prestación efectiva de la vivienda adecuada que esté al alcance de los hogares en estos países africanos.

Gopalan y Venkataraman (2015) abordan el problema de la vivienda adecuada en la India y argumentan cómo las decisiones de vivienda impactan diferentes esferas de la vida de los hogares. Establecen definiciones de vivienda adecuada en India y en el ámbito mundial, contrastando los problemas que existen con varias de las definiciones. El papel que desempeña y las funcionalidades de la vivienda son multifacéticas, en el sentido de que las decisiones de vivienda impactan el acceso al empleo, la infraestructura, la salud, la educación, los niveles de pobreza, entre otros. Trabajos como los de Mueller y Tighe (2007) han presentado evidencia de la relación entre la vivienda adecuada y los beneficios en educación y salud para las comunidades.

Por su parte, Dufty-Jones (2015) lleva a cabo una investigación analítica acerca de cómo la movilidad, vista como uno de los principales ejes, impacta el consumo de vivienda rural en Australia; de hecho, su política ha sido poco estudiada en el marco internacional con fines de conocer las relaciones entre movilidad y vivienda rural. En este trabajo, el autor establece un análisis de casos en Australia relacionados con la política de vivienda rural y regional, entre 1985 y 2000. Los resultados de la evaluación crítica que hace el autor muestran que durante estos años la movilidad fue una política racional de carácter gubernamental, basada en la información de las políticas que se implementaban en cada región.

En un contexto multirregiones, Ward y Smith (2015) exploran algunas formas en que las aplicaciones de políticas de vivienda sostenible pueden extenderse a todos los segmentos del mercado de vivienda, incluso a los de menos ingresos. La idea de los autores es que tanto los diseñadores de las políticas como los hogares propietarios de bajos ingresos puedan participar conjuntamente en la construcción de viviendas sostenibles. Las experiencias en América Latina han sido exitosas, principalmente en el tema de rehabilitación de la vivienda.

Por último, Shimbo (2012) permite analizar los límites de la política de vivienda adecuada y asequible, y contrasta la llamada política habitacional con el interés de grandes empresas constructoras en el tamaño de la producción de viviendas. Estos análisis evidencian una gran preocupación con respecto al modelo de ciudades que estamos construyendo, en términos de geografía, urbanismo, arquitectura, sociología y planeación urbana.

\section{ANTECEDENTES DE LA POLÍTICA DE VIVIENDA Y EL SECTOR FINANCIERO EN COLOMBIA}

La política pública de vivienda y el sistema financiero en Colombia han estado vinculados estrechamente en la historia del país ${ }^{1}$. Los orígenes de este fuerte vínculo se remontan a los años precedentes a 1972, que se caracterizaron por la captación de recursos por parte de compañías de seguros y bancos comerciales, al igual que por su canalización hacia el sector de la construcción por medio del Banco Central Hipotecario ( $\mathrm{BCH}$ ) y el Instituto de

1 Para una revisión más exhaustiva de las reformas financieras acaecidas en el país, en torno a la política de vivienda, se recomienda consultar a Jaramillo (2009), investigador que ha estudiado a fondo la problemática y cuenta con numerosos trabajos respecto al tema, particularmente en Bogotá. 
Crédito Territorial $(\mathrm{ICT})^{2}$. Otras opciones de financiación de vivienda eran los ahorros personales, los créditos bancarios a corto plazo y los subsidios; sin embargo, el aporte del sector de la construcción era bastante bajo en el crecimiento y persistían altos costos para el sector público.

A finales de 1972, un novedoso sistema entró en vigencia en el mercado de vivienda colombiano bajo los decretos 677, 678 y 1229 de dicho año. El sistema conocido como Unidad de Poder Adquisitivo Constante (UPAC) buscaba mantener el poder adquisitivo de la moneda y ofrecer a los colombianos la opción de obtener un crédito de largo plazo para comprar vivienda. Así se garantizaba que el dinero captado y prestado se contabilizara en unidades que se actualizarían con el comportamiento de la inflación, y de esta manera los ahorradores no perderían poder de compra, en tanto los prestatarios no recibirían bajos intereses por sus préstamos de largo plazo (Echeverri, García y Pineda, 1999).

En principio, el sistema UPAC incentivó el ahorro, en tanto las corporaciones tuvieron el dinero suficiente para prestar grandes cantidades a los constructores y así dinamizar el sector. De esta forma se incentivó la construcción de viviendas nuevas y usadas, la construcción de fábricas y edificaciones urbanas, y se generó empleo de mano de obra no calificada.

Por su parte, el ICT era el organismo estatal que tenía como función producir vivienda para los sectores más favorecidos, asignar subsidios y otorgar créditos de largo plazo con precios y tasas mucho más bajas. Otro organismo promotor importante fue la Caja de Vivienda Popular (CVP) ${ }^{3}$,

$2 \quad$ El BCH fue un banco estatal creado en 1932 con el objetivo de canalizar los recursos de crédito para vivienda. Fue liquidado en el 2000, debido a la grave crisis financiera. EI ICT fue creado en 1939 para ejecutar funciones de construcción y financiación de vivienda de interés social (VIS); en 1991 se convirtió en el Instituto Nacional de Vivienda de Interés Social y Reforma Urbana (INURBE), cuya función principal era la adjudicación de subsidios para mejoramiento, construcción y adquisición de VIS.

3 Actualmente, la Caja de Vivienda Popular ejecuta políticas en programas de mejoramiento de barrios, de vivienda, titulación, urbanizaciones y reasentamientos urbanos, con que fue de suma importancia, por ejemplo, en la construcción de los proyectos de Ciudad Kennedy y Ciudad Bolívar, que albergan parte significativa de la población capitalina (Jaramillo, 1992).

Hacia 1982 nació la preocupación por las llamadas viviendas de interés social (VIS). La administración de Belisario Betancourt pretendía que los constructores privados participaran en dicho segmento. Fue entonces cuando el Gobierno creó el plan de vivienda sin cuota inicial para los sectores con más bajos recursos; sin embargo, la rentabilidad para el sector privado, bajo este plan, no era comparable con la rentabilidad que se percibía en la construcción de vivienda no VIS, de ahí que no tuvo suficiente impacto (Jaramillo, 1992).

En 1989 se reglamentó lo que se conoció como Reforma Urbana, que otorgaba subsidios para la financiación de VIS y que optó por dar créditos, para este tipo de vivienda, a través de la UPAC. El gobierno tomó los depósitos del sistema UPAC que los bancos no querían prestar y los destinó a la VIS. Esto trajo consecuencias negativas para el ICT, puesto que su cartera morosa incrementó. Aunque los sectores público y privado llegaron a un acuerdo por generar vivienda VIS y mantener un volumen apropiado de construcción de esta, el ICT fue considerado ineficaz y se planteó la necesidad de reemplazarlo.

La UPAC siguió funcionando de forma similar, y para muchos fue un exitoso sistema que incentivó la construcción de vivienda en el país; sin embargo, el afán por hacer de la UPAC un sistema más competitivo llevó a realizar en él cambios cada vez más drásticos: desde 1990 fue incrementando la participación de la tasa variable DTF, calculada por el Banco de la República para el mes anterior en la composición del sistema UPAC, hasta que en 1995 dependía en su totalidad de la DTF (tabla 1).

También, desde 1990 se reformó el sistema financiero y se abandonaron las medidas proteccionistas a los bancos, se promovió la libre competencia y todas las entidades bancarias podían ofrecer

el propósito de mejorar la calidad de vida en los estratos 1 у 2. 
Tabla 1.

Reformas al sistema UPAC

\begin{tabular}{|c|c|}
\hline Año & Cálculo del sistema \\
\hline 1973 & IPC promedio de los 12 meses inmediatamente anteriores. \\
\hline 1974 & $\begin{array}{l}\text { IPC promedio de los } 24 \text { meses inmediatamente anteriores con un límite al aumento de su valor } \\
\text { del } 20 \% \text { anual. }\end{array}$ \\
\hline 1975 & $\begin{array}{l}\text { IPC promedio de los } 24 \text { meses inmediatamente anteriores con un límite al aumento de su valor } \\
\text { del } 19 \% \text { anual. }\end{array}$ \\
\hline 1976 & $\begin{array}{c}\text { Con el decreto } 58 \text { el sistema se componía por el promedio del IPC de los } 12 \text { meses inmediatamente } \\
\text { anteriores con un límite al aumento de su valor del } 18 \% \text { anual. }\end{array}$ \\
\hline 1982 & $\begin{array}{c}\text { Con el decreto } 2929 \text { el sistema se componía por el promedio del IPC de los } 12 \text { meses inmediatamente } \\
\text { anteriores con un límite al aumento de su valor de } 23 \% \text { anual. }\end{array}$ \\
\hline 1988 & $\begin{array}{l}\text { Con el decreto } 1319 \text { la DTF (Deposito a Termino Fijo) empieza a formar parte de su composición. Al } 40 \% \\
\text { de la variación del IPC de los } 12 \text { meses anteriores se le adiciona el } 35 \% \text { del promedio de la DTF. Su límite } \\
\text { era el } 24 \% \text { anual. }\end{array}$ \\
\hline 1990 & $\begin{array}{l}\text { Con el decreto 1127, al } 45 \% \text { de la variación resultante del IPC para los } 12 \text { meses anteriores se le adiciona } \\
\text { el 35\% del promedio de la DTF publicada para el mes anterior. }\end{array}$ \\
\hline 1992 & $\begin{array}{l}\text { El 20\% de la variación resultante del IPC para } 12 \text { meses anteriores se le adiciona el } 50 \% \text { del promedio } \\
\text { de la DTF publicada para el mes anterior. }\end{array}$ \\
\hline 1995 & Depende en su totalidad de la DFT. \\
\hline
\end{tabular}

Fuente: elaboración de los autores a partir de Jaramillo (2009).

diversos productos financieros. Un año más tarde se reformó el sistema de interés social, contexto en que el Estado abandonó su papel de intermediario financiero y pasó a ser Estado subsidiario. Para ello se creó el Instituto Nacional de Vivienda de Interés Social y Reforma Urbana (INURBE), que se encargaba de regular y otorgar los subsidios a las personas que más lo necesitaran. Tras la búsqueda de la eficiencia se crearon más adelante las cajas de compensación, que desempeñarían un papel fundamental en la asignación de estos subsidios para empleados de bajos ingresos y sus familias, y a su vez actuaran como promotoras de vivienda.

Como se mostrará más adelante, en los primeros años de la década de los noventa, el auge económico había jalonado el crecimiento del sector de la construcción; sin embargo, el panorama favorable cambió cuando la DTF empezó a crecer más que el IPC. Puesto que a las personas que tenían sus créditos bajo el sistema UPAC les eran cobradas cuotas de su crédito hipotecario muy superiores a su capacidad de pago, muchos propietarios optaron por devolver sus viviendas, con lo cual se afectó directamente el sector de la construcción, el sistema de créditos de vivienda y el crecimiento económico.

Tras el colapso de la UPAC en 1999 el Congreso de la República estableció la Unidad de Valor Real (UVR), que al igual que la UPAC, tiene como objetivo realizar una corrección monetaria y ajustar el valor de los créditos de acuerdo con el costo de vida del país. Su evolución está ligada a la evolución del IPC y es certificada por el Banco de la República, por lo que más que un cambio en el sistema, se retornó a la idea que concibió la UPAC. La gráfica 1 ilustra la evolución de ambas unidades.

En cuanto al entorno crediticio reciente, el comportamiento macroeconómico de los últimos años, la inflación controlada (IPC), las consecuentes bajas tasas de interés y los estímulos por medio de subsidios y flexibilización de requisitos han permitido que una mayor proporción de la población 
Gráfica 1 .

Variación anual de la UPAC y de la UVR durante su existencia.
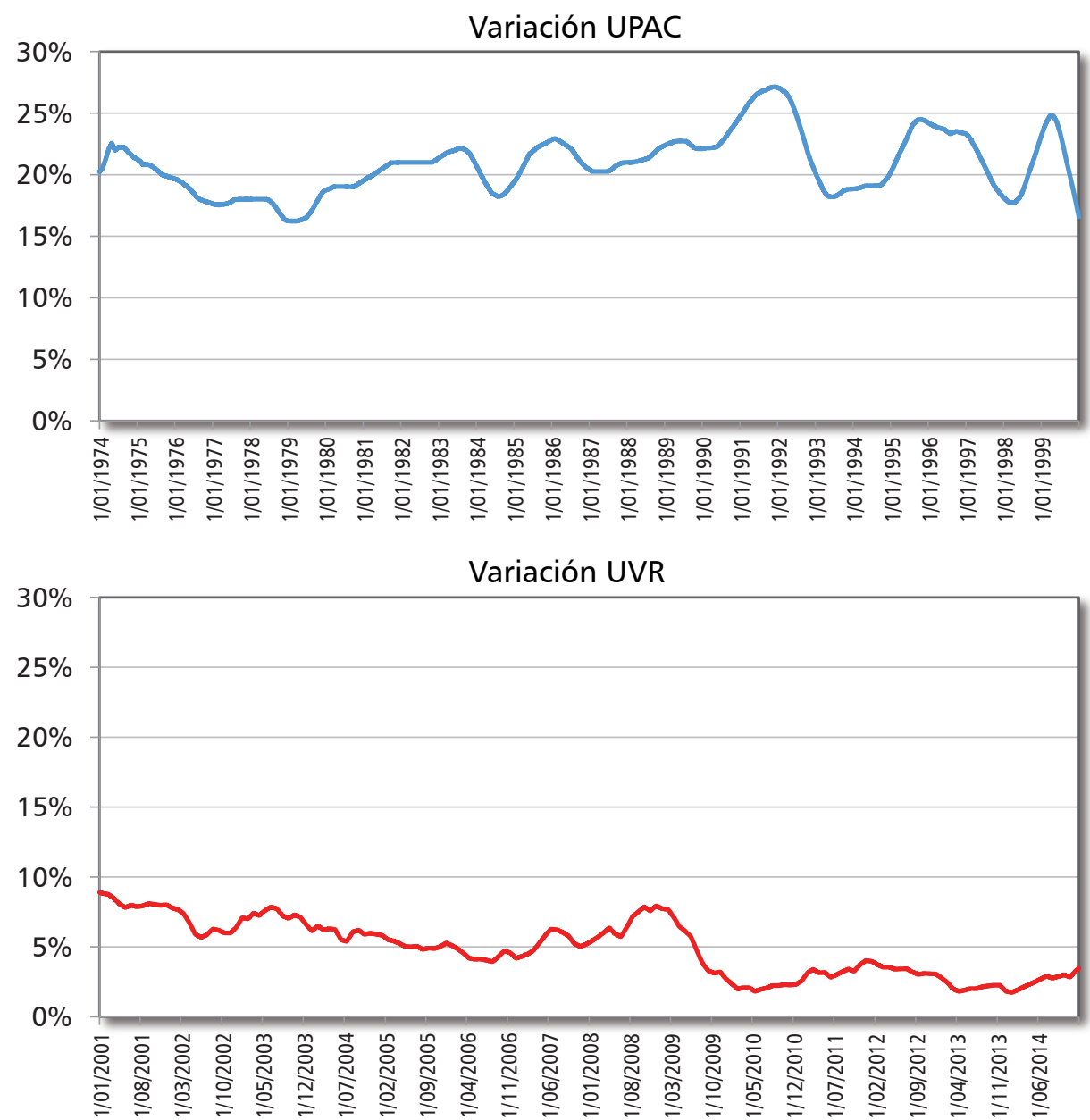

Fuente: Banco de la República.

logre acceder a créditos de vivienda. Por ejemplo, en 2009, como consecuencia de la crisis financiera internacional, el Gobierno nacional promovió la política de subsidios a la tasa de interés, consistente en la cobertura de la tasa durante los primeros años del crédito. Se trata de una política contracíclica que buscaba incentivar la demanda, lo que, según Urrutia y Namen (2012), funcionó.

Como se observa en la tabla 2, el número de créditos hipotecarios creció significativamente entre 2005 y 2014, especialmente en el caso de beneficiarios de VIS. Por su parte, el capital con cuotas vencidas disminuyó en ambos rangos de vivienda. Se destaca la importancia de la vivienda VIS, en cuanto al número de créditos hipotecarios, que representan cerca del $55 \%$ del total en el primer trimestre de 2014, mientras que el capital con cuotas vencidas representó el $36 \%$. No obstante, la participación VIS en el capital con cuotas vencidas ha crecido levemente (gráfica 2), lo que podría agravarse en épocas de recesión económica. 
Créditos hipotecarios y capital con cuotas vencidas según tipo de vivienda

\begin{tabular}{|c|c|c|c|c|}
\hline \multirow{2}{*}{ Periodo } & \multicolumn{2}{|c|}{ Número de créditos hipotecarios } & \multicolumn{2}{c|}{$\begin{array}{c}\text { Capital con cuotas vencidas } \\
\text { (millones de \$ de 2005*) }\end{array}$} \\
\hline & VIS & No VIS & VIS & No VIS \\
\hline $2005-I$ & 393.135 & 381.938 & 281.953 & 831.623 \\
\hline $2014-I$ & 507.508 & 425.520 & 265.909 & 474.589 \\
\hline
\end{tabular}

Fuente: Banco de la República y cálculos propios*.

Gráfica 2.

Distribución de créditos hipotecarios y cuotas vencidas según tipo de vivienda (2005-IV a 2014-IV)

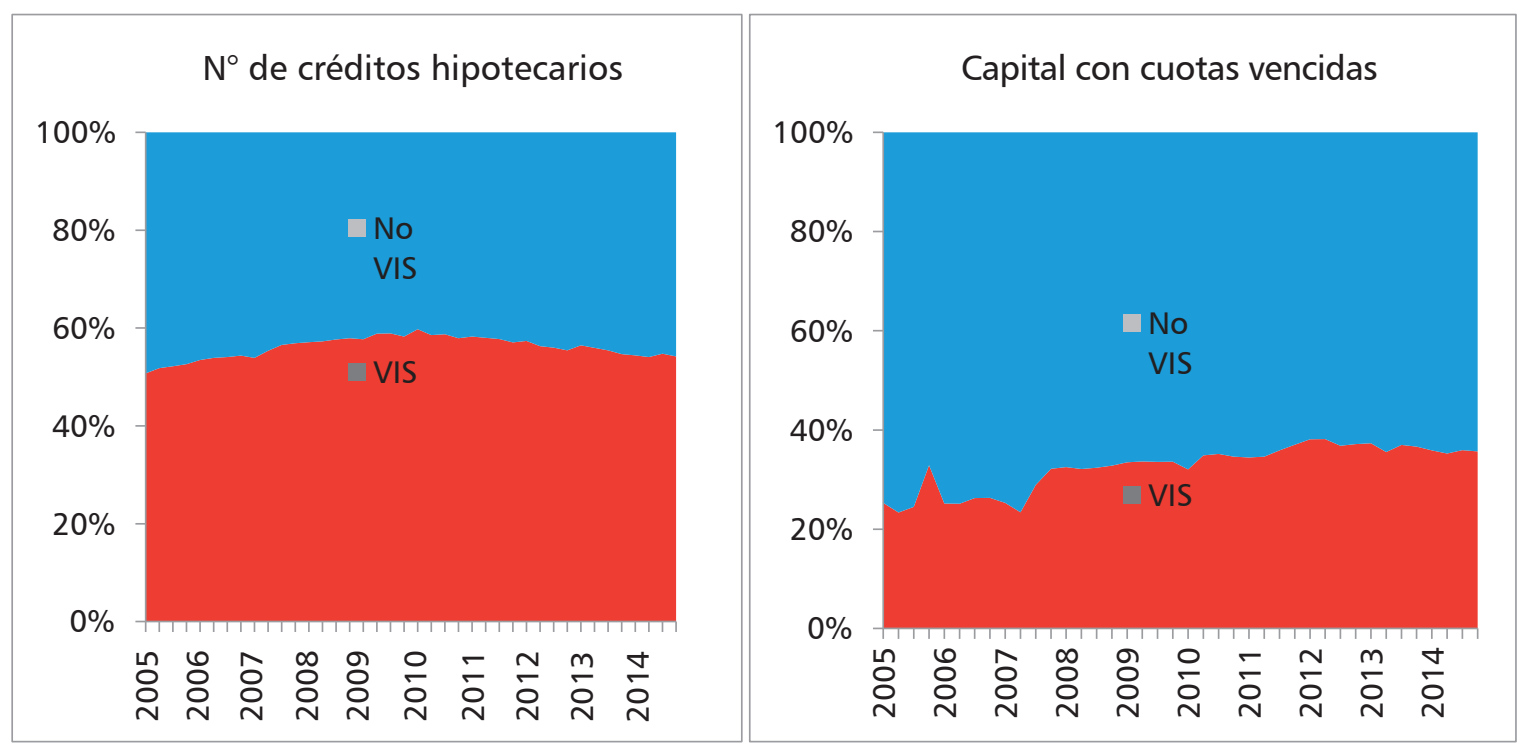

Fuente: Departamento Administrativo Nacional de Estadística (DANE), Cuentas Nacionales.

\section{DESARROLLO URBANO Y EL SECTOR DE LA CONSTRUCCIÓN EN BOGOTÁ DURANTE LAS ÚLTIMAS DÉCADAS}

Desde la década de los setenta, la población de Bogotá comenzó a crecer rápidamente, resultado de fenómenos como el desarrollo industrial, la violencia rural y el mejoramiento de la infraestructura vial y de comunicaciones en el país; sin embargo, dicho crecimiento se desarrolló de manera desordenada y careció de una planificación urbana de largo plazo. Los problemas de hábitat en Bogotá y las medidas adoptadas durante este proceso comenzaron a ser estudiados por la academia y las instituciones gubernamentales desde la década de los ochenta, cuando ya se evidenciaba el surgimiento del sector informal en vivienda ${ }^{4} y$ un desplazamiento masivo de personas de otras regiones hacia Bogotá (Villamizar, 1989; Jaramillo y Cuervo, 1987; Jaramillo, 1992).

$4 \quad$ Se puede entender el sector informal en vivienda como el surgimiento de lo que se conoce como urbanizaciones piratas e ilegales. Este argumento es desarrollado por Brucher y Mertins (1978) y, más recientemente, por Vergara (2008). 
A pesar de las particularidades sociales y políticas que enfrentó el país, la problemática no era muy distinta en otros países de la región. Grandes ciudades como Ciudad de México, Río de Janeiro y Lima también experimentaron un incremento en el número de urbanizaciones ilegales, caracterizadas por emerger en zonas periféricas de la ciudad y con poblaciones de bajos recursos. Esto constituye uno de los factores en los que se funda la problemática de segregación social y exclusión en las ciudades, esto es, la fragmentación de la sociedad espacial y socialmente (Borja, 2001).

En Colombia, específicamente en Bogotá, el papel del Estado se ha enfocado en la provisión de vivienda nueva, pero relegando en gran medida las políticas de mejoramiento de los barrios y la ciudad construida (Vergara, 2008) e impulsando aún más el crecimiento horizontal de esta. Así, la política pública, desde sus inicios, no se preocupó necesariamente por el uso eficiente del suelo y limitó la posibilidad de una planificación adecuada y sostenible del proceso de urbanización en la ciudad. Esto, sin embargo, no significa la ausencia de una política de vivienda para la ciudad, sino una orientación casi estricta a lograr coberturas que redujesen el déficit cuantitativo, sin prever fenómenos sociodemográficos como los acaecidos en la ciudad en las últimas décadas.

Un aspecto destacable en la política de vivienda fue el estímulo permanente al sector de la construcción. Como es habitual en la ejecución de la política económica, esta se dirigió a favorecer la economía en el corto plazo; allí el sector de la construcción fue un importante impulsor del crecimiento, el empleo y la demanda agregada. Como se anotó, el sector financiero desempeñó un papel determinante en la promoción de mecanismos de financiación de vivienda nueva, así como el estímulo al consumo hipotecario, que redundó en un crecimiento importante del sector hasta finales de la década de los ochenta (gráfica 3). Es así como la política evidenció una preocupación cortoplacista que ignoraba los problemas subsecuentes ya mencionados.

La gráfica 3 indica un comportamiento contracíclico del sector de la construcción hasta principios de la década de los noventa; los periodos de alto crecimiento económico se asociaron

Crecimiento del PIB en el sector construcción vs. total (1976-2013)

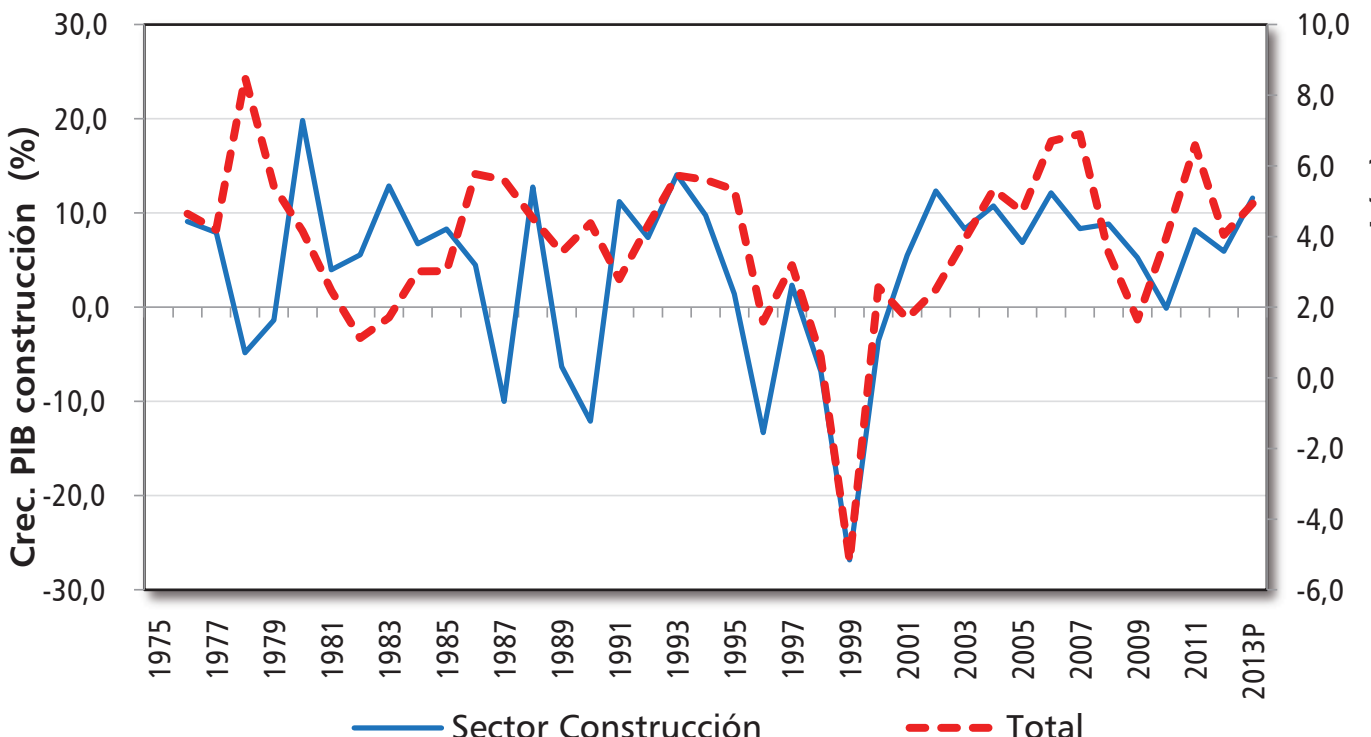

Fuente: Departamento Administrativo Nacional de Estadística (DANE), Cuentas Nacionales. 
con caídas en el sector de la construcción. Dicha caída es particularmente notoria en 1978 (-4,8\%), precisamente cuando la economía colombiana presentó tasas de crecimiento de $8,5 \%$, las más altas de su historia, fruto, entre otros elementos, de la política de sustitución de importaciones y la bonanza cafetera.

Asimismo, la política fiscal expansiva, efectuada por la administración de Julio César Turbay (1978-1982), significó mayor contratación en el sector de la construcción, que acompañó un crecimiento del sector cercano al $20 \%$ en 1980 , mientras que el resto de la economía se deterioró, debido a la caída de los precios del café y, poco después, a la crisis financiera que enfrentó la región.

El inicio de la década de los noventa se caracterizó por la serie de reformas estructurales dirigidas a liberalizar la economía, de modo que la estructura productiva y sectorial, así como la evolución de la demanda agregada, comenzaron a transformarse significativamente. El sector construcción dejó de concebirse como un instrumento de política contracíclica, como aparentemente lo fue, y comenzó a responder a los estímulos de la actividad económica en general.

Hacia 1993 comienza a manifestarse una nueva dinámica y se vislumbra la recuperación económica, pero dicho impulso se agotaría rápidamente tras la desaceleración de 1994 y 1995, tanto en el sector construcción como en la economía en general. La crisis llegaría a su culmen en 1999, con caídas de $5 \%$ en el PIB total y de $27 \%$ en el PIB del sector construcción.

De la estrecha relación entre el sector de la construcción y el PIB total de la economía se destaca un resultado: el mercado de la vivienda en las últimas dos décadas ha estado sujeto al ciclo económico. Ahora bien, es necesario reconocer que el sector de la construcción no refleja en su totalidad las condiciones del mercado de vivienda, pues refleja esencialmente condiciones de oferta de vivienda. El índice de precios de la vivienda, conjuntamente, permite inferir aspectos desde la demanda ${ }^{5}$.

La gráfica 4 muestra cuatro índices: a) el índice de precios de apartamentos nuevos, b) el índice de precios de casas nuevas, c) el índice de precios de vivienda usada (casas y apartamentos) y c) el índice de costos de construcción de vivienda; todos en términos reales ${ }^{6}$.

Los índices reales permiten aislar el efecto de la inflación del resto de la economía, para lo cual concentran la atención en la variación "real" del precio de la vivienda. A pesar de que durante la última década los costos de la construcción se han venido reduciendo o, por lo menos, han sido estables en términos reales, los precios de la vivienda han crecido de manera muy acelerada. Este resultado apoya la hipótesis de algunos observadores: el mercado de la vivienda ha experimentado en los últimos años una burbuja de demanda (Piraquive y Hernández, 2014; Salazar et al., 2015).

La vivienda usada presenta el escenario más notable. De acuerdo con los datos del Banco de la República, los precios de este rango alcanzaron un alza del $137 \%$ entre 2006 y 2014 en el ámbito nacional. De acuerdo con la gráfica 4, esto equivale a una variación cercana al $76 \%$ en términos reales. La tendencia en Bogotá es similar pero más acelerada, con incrementos reales en los precios superiores a $80 \%$ en ese mismo periodo, tanto para vivienda nueva como usada. Se puede corroborar, además, que si se consideran los últimos diez años,

$5 \quad$ Los aspectos asociados a la demanda se concentran en dos aspectos: el primero, los estímulos que desde el sector financiero se realiza a los hogares para la compra de vivienda; y el segundo, el ritmo de la actividad económica, manifestado principalmente en el crecimiento del PIB y el nivel de empleo. El índice de precios de vivienda nueva (casas y apartamentos) es elaborado por el Banco de la República y consiste en un promedio simple del precio por metro cuadrado de todos los inmuebles nuevos; de ahí que no hace consideraciones de calidad. El índice de precios de la vivienda usada también lo calcula el Banco de la República, basado en la metodología descrita en Escobar et al. (2005). El índice de costos de construcción es calculado por el DANE. Los índices reales se calcularon corrigiendo por el IPC total y el IPC de Bogotá, según el caso. 
los precios de la vivienda han crecido aproximadamente el doble de lo que se han incrementado en el resto de los precios de la economía?.

Gráfica 4.

Índices reales de precios de la vivienda en el ámbito nacional y en Bogotá (1997-2014).
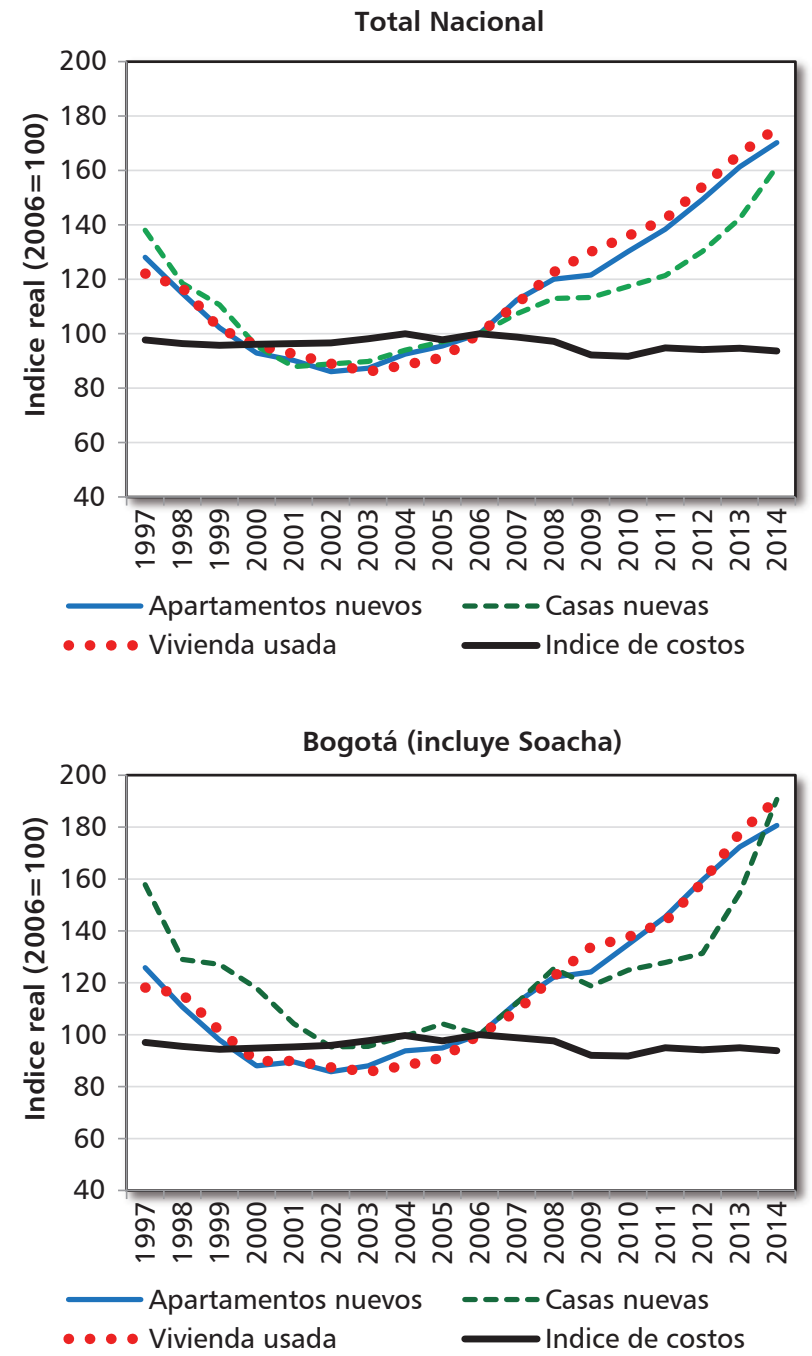

Fuente: DANE, Banco de la República y cálculos propios*.

Una aproximación a la composición de la vivienda en Bogotá permite vislumbrar algunos hechos recientes en torno al mercado, así como

$7 \quad$ En este documento no se afirma ni se determina rigurosamente la existencia de una burbuja inmobiliaria en el país: sin embargo, algunos trabajos corroboran que las dinámicas mostradas aquí sugieren la existencia de dicha burbuja (Gómez et al., 2013; Franco et al., 2014). marcar algunos elementos inherentes a la problemática actual. La tabla 3 presenta información sobre la evolución del área en construcción según destino de uso en Bogotá.

En primer lugar, la composición del área en construcción no ha tenido cambios importantes en los últimos años en términos de su destino; el área más significativa es la destinada a apartamentos, que representa entre el $59 \%$ y el $63 \%$ en los últimos años. En segundo lugar, se destaca el constante aumento en el área en construcción: pasó de $5.169 .318 \mathrm{~m}^{2}$ en el cuarto trimestre de 2006, a $6.220 .418 \mathrm{~m}^{2}$ en el mismo trimestre de 2010, y a $7.806 .735 \mathrm{~m}^{2}$ en el 2014 .

Finalmente, el argumento del agotamiento de la tierra disponible para construcción, si bien es difícil de cuestionar, debe ser considerado a la luz de los datos. El incremento en el área en construcción sugiere que la oferta sigue creciendo, lo que puede deberse a dos factores:

a) El mayor aprovechamiento del terreno disponible mediante la construcción de edificios. Es notorio que el crecimiento en el área en construcción se ha dado en apartamentos, oficinas, comercio, hoteles, hospitales y administración pública; usos que se dan en mayor medida en edificaciones de mayor altura, de ahí que implican un mayor aprovechamiento del suelo. El área en construcción destinada a casas y bodegas ha disminuido en el periodo, hecho consistente con la escasez de suelo.

b) El surgimiento de nuevos proyectos de vivienda en las periferias de la ciudad. Recientemente han surgido proyectos de gran envergadura que generan una construcción masiva, fomentados en gran medida por las nuevas políticas de vivienda del Gobierno nacional desde el 2011. En este orden de ideas, es presumible que la problemática de crecimiento horizontal sea agravada por las políticas recientes. 
Tabla 3.

Evolución del área en construcción en la ciudad de Bogotá según destinos

\begin{tabular}{|c|c|c|c|c|c|c|}
\hline & \multicolumn{2}{|c|}{$2006-\mathrm{IV}$} & \multicolumn{2}{c|}{$2010-\mathrm{IV}$} & \multicolumn{2}{c|}{ 2010-IV } \\
\hline Destinos & $\mathrm{M}^{2}$ & $\%$ total & $\mathrm{M}^{2}$ & $\%$ total & $\mathrm{M}^{2}$ & \% total \\
\hline Apartamentos & 3.233 .218 & 63 & 3.936 .402 & 63 & 4.592 .638 & 59 \\
\hline Casas & 726.685 & 14 & 469.230 & 8 & 218.707 & 3 \\
\hline Oficinas & 246.320 & 5 & 559.736 & 9 & 1.242 .625 & 16 \\
\hline Comercio & 334.363 & 6 & 450.560 & 7 & 562.982 & 7 \\
\hline Bodegas & 159.873 & 3 & 141.631 & 2 & 80.313 & 1 \\
\hline Educación & 307.146 & 6 & 162.706 & 3 & 247.585 & 3 \\
\hline Hoteles & 18.056 & 0 & 187.829 & 3 & 232.039 & 3 \\
\hline Hospitales & 43.033 & 1 & 103.206 & 2 & 142.690 & 2 \\
\hline Administración pública & 32.147 & 1 & 92.476 & 1 & 125.796 & 2 \\
\hline Otros & 68.477 & 1 & 116.642 & 2 & 361.360 & 5 \\
\hline Total & 5.169 .318 & 100 & 6.220 .418 & 100 & 7.806 .735 & 100 \\
\hline
\end{tabular}

Fuente: DANE, Censo de edificaciones.

\section{ANÁLISIS DEL MERCADO DE VIVIENDA EN BOGOTÁ A PARTIR DE LA LEY DE OFERTA Y DEMANDA}

En las secciones anteriores se ha mostrado, por un lado, que las políticas de vivienda han sido generalmente enmarcadas en el acceso al financiamiento de vivienda $y$, por otro, que el sector de la construcción es dinámico y estrechamente ligado a la actividad económica en general. Toda esta revisión es útil para comprender las causas de la problemática actual del mercado, traducida, entre otros factores, en el incremento desmedido de los precios de la vivienda, la escasez del suelo en Bogotá y la expansión horizontal desordenada de la ciudad. Dichos factores generan segregación social y limitan el acceso a servicios sociales, culturales y laborales para un amplio segmento de la población; estos últimos elementos son centrales dentro del concepto de vivienda digna.

El alcance de esta sección es proveer una explicación simple, basada en la ley de la oferta y demanda, sobre los factores que han afectado al mercado y sobre la ineficacia de las políticas establecidas en los últimos años.

\section{Factores de oferta y demanda}

En primer lugar, realizamos un análisis de los factores de oferta y los factores de demanda. Los primeros hacen referencia a problemas de índole estructural, es decir, de largo plazo y que resultan de condiciones propias del bien en cuestión (la vivienda) o de fenómenos históricos que repercuten en la disponibilidad de vivienda actual. Y los factores de demanda se refieren a problemas de corto plazo y de decisiones de consumo de vivienda por parte de los hogares (más allá de su disponibilidad). Como se vislumbró en las secciones anteriores, los incentivos a la demanda han predominado sobre los incentivos a la oferta en Bogotá, principalmente porque los segundos son más difíciles de efectuar y generalmente no son alcanzables a corto plazo.

La figura 1 ilustra los principales problemas del mercado y constituye un diagnóstico útil para avanzar en propuestas de política que redunden en el bienestar de la población, especialmente de bajos recursos.

A partir de revisiones documentales, hechos estilizados y la evidencia factual desarrollada anteriormente, se destacan los siguientes factores de oferta: 
Factores de oferta y demanda que afectan el mercado de la vivienda

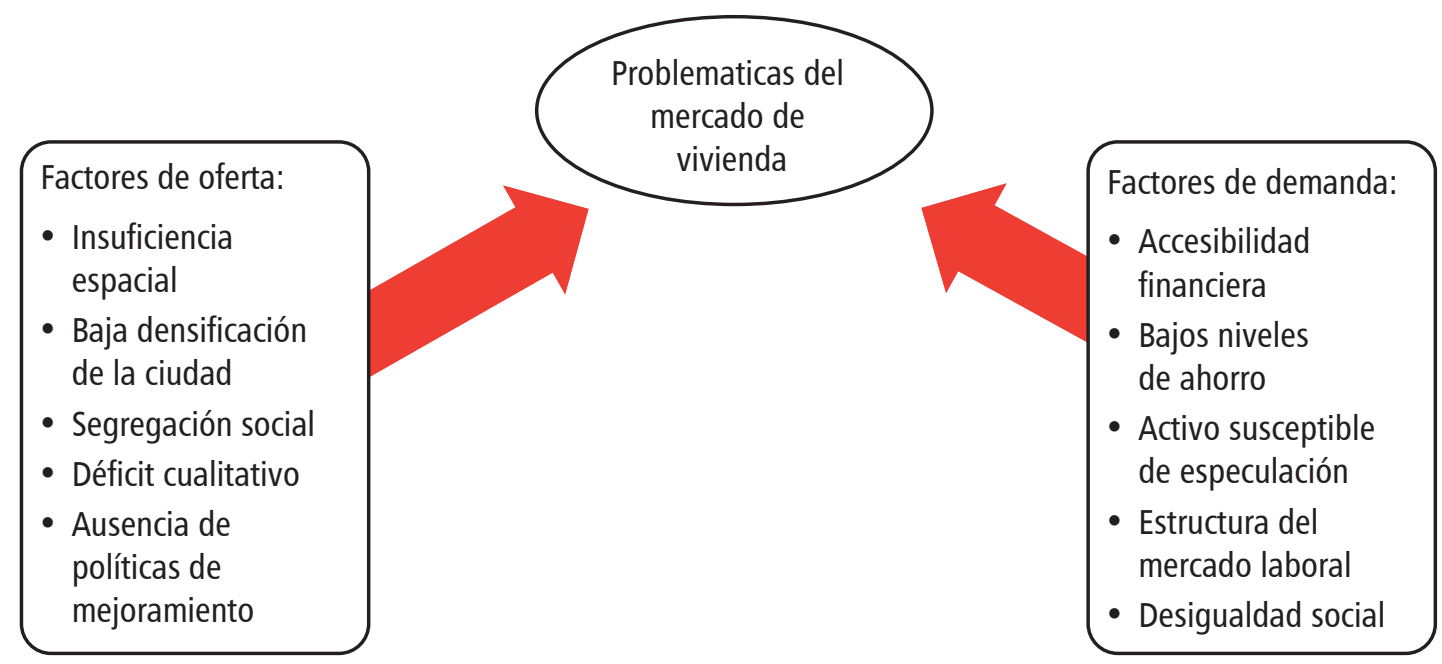

Fuente: elaboración de los autores.

a) Insuficiencia espacial. El crecimiento poblacional en Bogotá rebasa su infraestructura urbana, lo que se traduce en un déficit cuantitativo cada vez más difícil de superar. Desde un concepto de mercado, la oferta de tierra es altamente inelástica y las posibilidades de construcción de vivienda nueva se agotan.

b) Baja densificación de la ciudad. El desarrollo urbano no planificado de la ciudad ha generado un crecimiento espacial horizontal más que vertical, lo que hace ineficiente el uso de la tierra disponible. La baja densificación de la infraestructura trae consigo problemas de movilidad, costos de servicios públicos, infraestructura vial, marginalidad, entre otros.

c) Segregación social. La población bogotana no se encuentra integrada socialmente a causa de la marginalidad asociada a la ilegalidad de los barrios y la desigualdad en el acceso a los servicios públicos y privados de la ciudad. Los barrios periféricos tienden a caracterizarse por población de bajos recursos y, por lo tanto, con limitación en el acceso a servicios (parques, centros comerciales, centros deportivos, bibliotecas, etc.).

d) Déficit cualitativo. Más allá del déficit cuantitativo en Bogotá, existe un problema apremiante en la calidad de vivienda tanto nueva como usada. La vivienda nueva se ha desarrollado bajo criterios de habitabilidad cada vez menos dignos, mientras que en la vivienda usada persisten problemas de seguridad, acceso, entre otros.

e) Ausencia de políticas de mejoramiento. Es común que la oferta de vivienda nueva sea resultado de las imperfecciones del mercado, en particular, del exceso de demanda causado por la distorsión generada por las políticas estatales. En este sentido se resalta el hecho de que la vivienda nueva no resuelve el problema de calidad; por el contrario, puede perpetuar la problemática al utilizar la tierra disponible ineficientemente. 
Como factores de demanda se destacan los siguientes:

a) Accesibilidad financiera. La financiarización de la sociedad ha implicado un estado patrimonialista, lo que ha generado trampas de pobreza en la sociedad. Aquellos que no logran acceder al sistema crediticio, encuentran en los asentamientos informales el camino para lograr acceder a la propiedad. Precisamente para este tipo de hogares, la proporción de los ingresos que deben destinar a gastos en vivienda tiende a ser mayor.

b) Bajos niveles de ahorro. De las tres fuentes de recursos para acceder a vivienda (o mejoramiento de esta), es decir, ahorro, subsidio y crédito, la más importante en los compradores de vivienda sigue siendo el crédito hipotecario, mientras que en una sociedad deseable debería ser el ahorro.

c) Propiedad y especulación. La vivienda se entiende como una inversión, y como tal, está sujeta a especulación financiera y a la conformación de carteles en el sector de la construcción (caso de constructoras y cementeras).

d) Condiciones del mercado laboral. La flexibilización laboral y las altas tasas de desempleo del país (la más alta de la región) limitan la posibilidad de ahorro, así como la capacidad de pago de la población.

e) Desigualdad social. La desigualdad social es un fenómeno estructural que genera segregación y la necesidad de implementar políticas progresivas, es decir, que tengan en cuenta la heterogeneidad de los hogares beneficiarios.

\section{El problema del equilibrio}

Las condiciones de oferta de vivienda determinan la dinámica reciente del mercado: las políticas de demanda no son efectivas si no se acompañan de políticas de oferta, mientras que las políticas de oferta pueden ser más efectivas en función de reducir el déficit cuantitativo y, más aún, el déficit cualitativo. Esto último se logra, por ejemplo, con políticas de mejoramiento de vivienda existente.

Para evaluar la efectividad de un tipo de política u otra, expresemos, sin pérdida de generalidad, las funciones de demanda y oferta de vivienda así:

$Q^{d}=\alpha P^{-e d}$

$Q^{o}=\gamma P^{e o}$

Donde $\alpha$ y $\gamma$ son parámetros que miden la intensidad de la demanda y la oferta, respectivamente, $P$ es el nivel de precios de la vivienda, ed es la elasticidad del precio de la demanda y eo es la elasticidad precio de la oferta de vivienda. El equilibrio de este mercado resulta en la siguiente solución:

$$
\begin{aligned}
& P=\left(\frac{\alpha}{\gamma}\right)^{\frac{1}{e d+e o}} \\
& Q=(\alpha)^{\frac{e o}{e d+e o}}(\gamma)^{\frac{e d}{e d+e o}}
\end{aligned}
$$

Dadas las condiciones naturales del mercado que estamos considerando, sabemos que eo es baja dada la escasez del suelo; en particular, consideremos el caso extremo de elasticidad de la oferta cercana a cero. Por lo tanto, siendo eo $\rightarrow 0$, la solución converge a:

$$
\begin{aligned}
& \lim _{e o \rightarrow 0} P=\left(\frac{\alpha}{\gamma}\right)^{\frac{1}{e d}} \\
& \lim _{e o \rightarrow 0} Q=\gamma
\end{aligned}
$$

En resumen, una política de demanda (incrementos en $\alpha$ ) en este mercado resulta en incremento en los precios, mas no en aumentos en la cantidad. En contraste, una política de oferta (incrementos en $\gamma$ ) es la única efectiva en términos de cantidad.

La gráfica 5 describe de manera simple las características del mercado. La oferta de vivienda se encuentra asociada a la disponibilidad de suelo, de ahí que se considere un bien de oferta inelástica, al menos a partir de cierto punto (por ejemplo, el punto $B$ ). Incrementos en el precio 
El efecto de una política de demanda en el mercado de vivienda

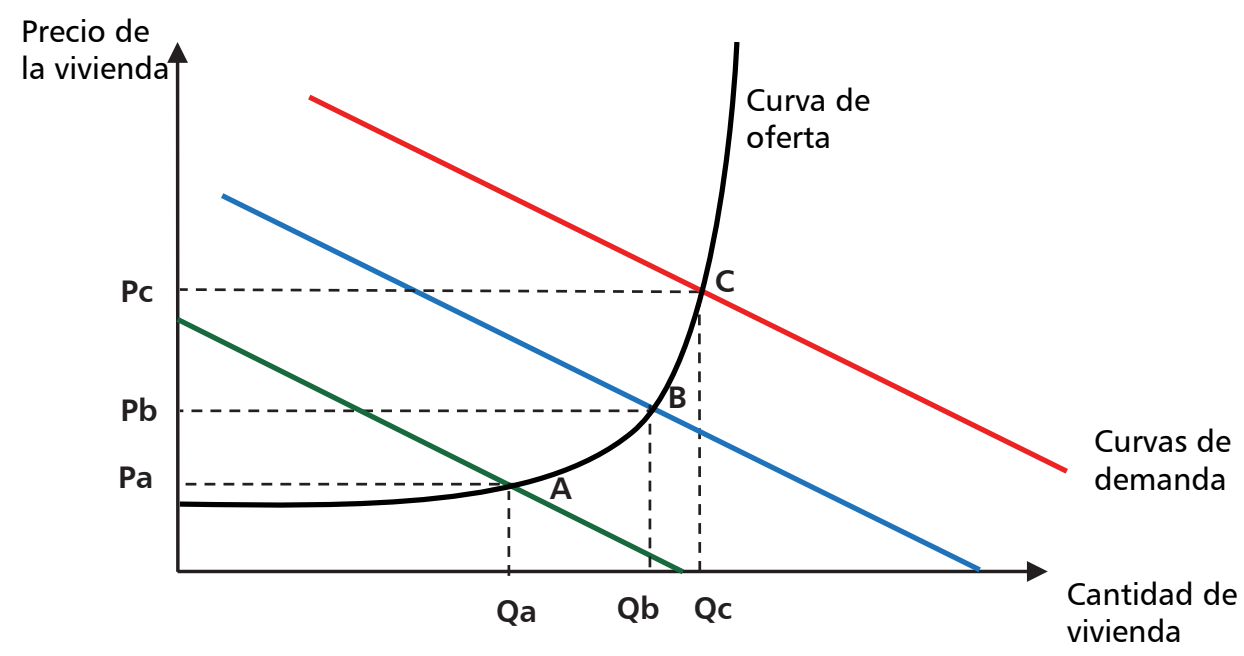

Fuente: elaboración de los autores.

no afectarán significativamente la disponibilidad del suelo.

Consideremos cambios en la curva de demanda, que pueden estar asociados a políticas de fomentos al crédito o subsidios a la tasa de interés. Un desplazamiento de la demanda del punto $A$ al punto $B$ implica mayores ingresos para los propietarios de la tierra, pues eleva los precios de esta; un desplazamiento de la demanda del punto $B$ al punto $C$ genera incrementos aún mayores en los precios, pero no afecta la cantidad de vivienda en el mercado. El paso del punto $A$ al $B$ caracteriza lo acaecido durante el siglo $\mathrm{XX}$, cuando la oferta de la tierra aún no era tan inelástica; el paso del punto $B$ al punto $C$ caracteriza lo ocurrido en las últimas dos décadas debido al agotamiento de la tierra y al uso ineficiente de la misma. Por lo tanto, una política de demanda en este contexto termina generando incremento en los precios, sin afectar significativamente la cantidad de vivienda y agravando la desigualdad del ingreso a favor de los oferentes.
La gráfica 6 ilustra el mismo mercado, en este caso caracterizando una política de oferta mediante el uso eficiente de la vivienda existente. Construir vivienda "hacia arriba" (mejoramiento de vivienda existente bajo criterios de eficiencia urbana) permite expandir la oferta de vivienda, al reducir los precios de esta, pero favoreciendo el ingreso de los propietarios del suelo (existente).

El agotamiento del suelo genera incremento permanente en los precios de la vivienda solo en la medida en que la ciudad sea construida exclusivamente de manera horizontal, como lo ha hecho históricamente; sin embargo, políticas de mejoramiento que propicien un desplazamiento de la oferta del punto $A$ al punto $B$ lograrían estabilizar (incluso reducir) los precios y mejorar las posibilidades de acceso a vivienda de la población. Al mismo tiempo, se generaría un ingreso efectivo (área sombreada) a los propietarios del suelo que participen en este tipo de políticas de mejoramiento, en particular, de construcción "hacia arriba". 


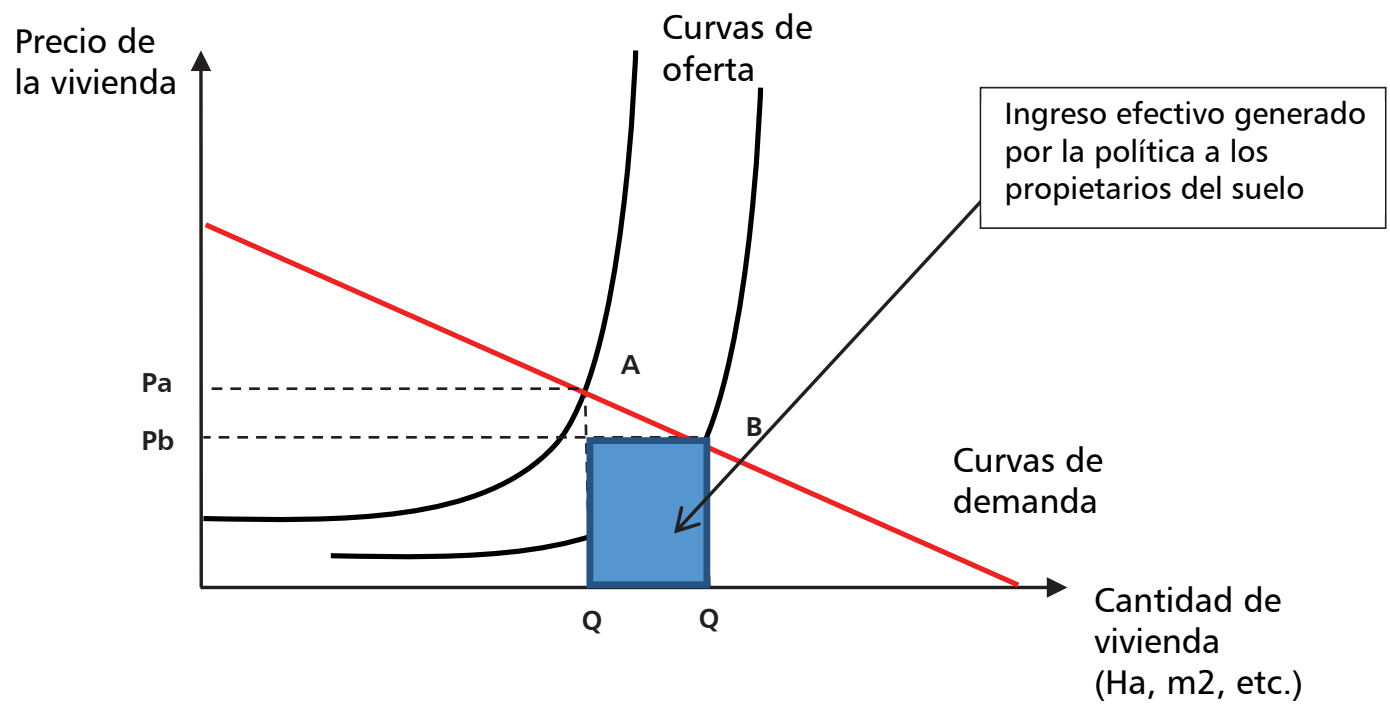

Fuente: elaboración de los autores.

\section{COMENTARIOS FINALES}

Durante muchos años, el papel del sistema financiero colombiano en las políticas gubernamentales ha permitido que una mayor proporción de la población logre acceder a vivienda propia; no obstante, el crecimiento poblacional y las dinámicas sociales emergidas en Bogotá han llevado a un desarrollo urbanístico carente de planificación. El surgimiento de barrios informales y en las periferias, con altos niveles de pobreza y poco acceso a servicios sociales, culturales y laborales, sugiere que el tipo de políticas en torno al sector deberían ser reconsideradas.

Dado lo anterior, este análisis defiende el argumento según el cual las políticas de demanda no suelen ser efectivas en el mercado de la vivienda, cuya prioridad debe pasar por garantizar niveles de vida dignas que sean sostenibles. El problema es que el mercado de la vivienda no se rige exactamente por la misma lógica de un mercado tradicional, no admite el mismo análisis que mercados de consumo masivo como los agrícolas, ni es posible asumir rendimientos a escala como en el caso de los bienes de contenido tecnológico o los servicios.

Si bien la vivienda se puede catalogar como un bien normal, presenta la particularidad, desde la demanda, de ser un bien prioritario cuyo consumo debe ser propiciado por políticas gubernamentales. Desde la oferta, los rendimientos son decrecientes debido al problema de congestión (Rubinfeld, 1987), particularmente en una sociedad altamente urbanizada como lo es Bogotá.

Las políticas de demanda basadas en subsidios a la vivienda y acceso al crédito hipotecario deben ser parte de un paquete de medidas contracíclicas, más que una política de largo plazo. La vivienda digna se construye a partir de criterios de calidad y acceso a servicios sociales que garanticen un crecimiento urbanístico sostenible para la ciudad. Los gobiernos suelen ser reacios a políticas de este tipo, debido a que su alcance es de largo plazo, por lo que sus resultados no son visibles en el gobierno de turno.

Finalmente, a pesar del crecimiento horizontal que ha tenido la ciudad, la evidencia muestra 
que Bogotá se está densificando mediante la mayor área destinada a apartamentos, oficinas y edificaciones altas. Si esta tendencia resulta de un proceso planificado y una política de mejoramiento basada en la construcción en sitio propio (Torres, García y Vargas, 2009), es más fácil propiciar condiciones sociales adecuadas a los habitantes y económicamente sostenibles. Los propietarios del suelo obtienen incentivos económicos mediante la valorización de sus activos, lo que constituye el método de financiamiento de este tipo de proyectos.

En contraste, Shimbo (2012) muestra una enorme preocupación con respecto al modelo de ciudades que estamos construyendo, en términos de geografía, urbanismo, arquitectura, sociología y planeación urbana. Debemos ser críticos y analizar si la política de vivienda es una política detrás del negocio de la construcción, o viceversa.

\section{REFERENCIAS}

1. Borja, J. (2001). El gobierno del territorio de las ciudades latinoamericanas. Revista Instituciones y Desarrollo, $8-9,83-142$

2. Brucher, W. y Mertins, G. (1978). Intraurbane Mobilität unterer Sozialschichten, randstädtische Elendsviertel und sozialer Wohnungsbau in Bogotá, Kolumbien. Marburger Geographische Schriften, 77, $1-130$.

3. Dufty-Jones, R. (2015). Governmentalities of mobility: The role of housing in the governance of Australian rural mobilities. Journal of Rural Studies, 42, 63-78.

4. Echeverri, J., García, O. y Pineda, B. (1999). UPAC: evolución y crisis de un modelo de desarrollo. Bogotá: Departamento Nacional de Planeación.

5. Escobar, J., Huertas, C., Mora, D. y Romero, J. (2005). Índice de Precios de la Vivienda Usada en Colombia (IPVU). Método de ventas repetidas. Bogotá: Banco de la República.

6. Franco, J., Gómez, J., Ojeda, J., Torres, E., (2014). Burbujas en precios de activos financieros: existencia, persistencia y migración. Bogotá: Banco de la República.

7. Gómez, J. et al. (2013). Testing for bubbles in housing markets: New results using a new method. Bogotá: Banco de la República.

8. Gopalan, K. y Venkataraman, M. (2015). Affordable housing: Policy and practice in India, IIMB Management Review, 27, 129-140.

9. Jaramillo, S. y Cuervo, L. M. (1987). La configuración del espacio regional en Colombia: tres ensayos. Bogotá: Centro de Estudios sobre el Desarrollo.

10. Jaramillo, S. y Cuervo, L. M. (2009). Dos décadas de política de vivienda en Bogotá apostando por el mercado. Bogotá: Centro de Estudios sobre el Desarrollo.

11. Jaramillo, S. (1992). La vivienda en Bogotá. Evolución de las condiciones habitacionales y de la estructura de producción de vivienda. Bogotá: Centro de Estudios sobre el Desarrollo.

12. Novoa, A., Bosch, J., Díaz, F., Malmusi, D., Darnell, M. y Trilla, C. (2014). El impacto de la crisis en la relación entre vivienda y salud. Políticas de buenas prácticas para reducir las desigualdades en salud asociadas con las condiciones de vivienda. Gaceta Sanitaria, 28(1), 44-50.

13. Mueller, E., y Tighe, J. (2007). Making the case for affordable housing: connecting housing with health and education outcomes. Journal of Planning Literature, 21 (4), 371-385.

14. Piraquive, G. y Hernández, G (2014). Evolución de los precios de la vivienda en Colombia. Bogotá: Departamento Nacional de Planeación. 
15. Portafolio.co (2013, 16 de junio). Ciudad Verde va acelerada. Recuperado de www.portafolio.co/negocios/ proyecto-ciudad-verde

16. Rubinfeld, D, (1987). The economics of the local public sector. Handbook of Public Economics, 2, 571-645.

17. Salazar, N., Steiner, R., Becerra, A. y Ramírez, J. (2015). Los efectos del precio del suelo sobre el precio de la vivienda para Colombia, Ensayos Sobre Política Económica, 31 (70), 17-66.

18. Secretaría de Planeación Distrital (2011). Primera encuesta multipropósito para Bogotá 2011. Bogotá: Autor.

19. Shimbo, L. (2012). Vivienda social de mercado: confluencia entre Estado, empresas constructoras y capital financiero. Toluca: Universidad Autónoma del Estado de México

20. Tipple, G. (2015). Housing policy-making in Africa: Ten common assumptions. Habitat International, 49, 413-418.

21. Torres, C., Rincón, J. y Vargas, J., (2009). Pobreza urbana y mejoramiento integral de barrios en Bogotá. Bogotá: Universidad Nacional de Colombia.

22. Urrutia, M. y Namen, O., (2012). Historia del credito hipotecario en Colombia. Ensayos sobre Política Económica, 30(67).

23. Vergara R., (2008). El mejoramiento de vivienda y entorno en Bogotá, 1994-1998: programas, conceptos y estrategias. Revista Investigación y Desarrollo, 16(1), 196-225.

24. Villamizar, R. (1980). Los precios de la tierra en Bogotá (1955-1978). Revista Cámara de Comercio de Bogotá, 11, 11-170.

25. Villaveces, J. (2007). La vivienda en la perspectiva de seguridad. Bogotá: Universidad del Rosario.

26. Ward, P. y Smith, C. (2015). Housing rehab for consolidated informal settlements: A new policy agenda for 2016 UN-Habitat III. Habitat International, 50, 373-384. 